\title{
Intraluminal EWSR1-CREB1 gene rearranged, low-grade myxoid sarcoma of the pulmonary artery resembling extraskeletal myxoid chondrosarcoma
} (EMC)

Opitz, Isabelle ; Lauk, Olivia ; Schneiter, Didier ; Ulrich, Silvia ; Maisano, Francesco ; Weder, Walter ; Bode-Lesniewska, Beata

\begin{abstract}
Fusions of the EWSR1 gene with partners from the CREB gene family have been identified in several histologic entities, mostly mesenchymal tumors of variable clinical malignant potential, including angiomatoid fibrous histiocytomas (AFH), clear cell sarcomas (CCS), clear cell sarcoma-like tumors of the gastrointestinal tract (CCSLTGT), primary pulmonary myxoid sarcoma (PPMS), intracranial myxoid neoplasias (iMN) and rare carcinoma variants. Interestingly, some of EWSR1-CREB-family gene fusion associated tumors show a prominent myxoid component and some of such tumors were histologically in the past diagnosed as "consistent with extraskeletal myxoid chondrosarcomas (EMC)".
\end{abstract}

DOI: https://doi.org/10.1111/his.13773

Posted at the Zurich Open Repository and Archive, University of Zurich ZORA URL: https://doi.org/10.5167/uzh-157530

Journal Article

Accepted Version

Originally published at:

Opitz, Isabelle; Lauk, Olivia; Schneiter, Didier; Ulrich, Silvia; Maisano, Francesco; Weder, Walter; Bode-Lesniewska, Beata (2019). Intraluminal EWSR1-CREB1 gene rearranged, low-grade myxoid sarcoma of the pulmonary artery resembling extraskeletal myxoid chondrosarcoma (EMC). Histopathology, 74(3):526-530.

DOI: https://doi.org/10.1111/his.13773 
PROFESSOR BEATA BODE-LESNIEWSKA (Orcid ID : 0000-0002-3329-4186)

Article type : Correspondence

\section{Intraluminal EWSR1-CREB1 gene rearranged, low-grade myxoid sarcoma of the pulmonary artery resembling extraskeletal myxoid chondrosarcoma (EMC)}

Running Title: EWSR1-CREB1 sarcoma of the pulmonary artery

Isabelle Opitz $^{1 \S}$, Olivia Lauk ${ }^{1 \S}$, Didier Schneiter ${ }^{1}$, Silvia Ulrich ${ }^{2}$, Francesco Maisano ${ }^{3}$, Walter Weder ${ }^{1}$, Beata Bode-Lesniewska ${ }^{4}$

${ }^{1}$ Department of Thoracic Surgery, University Hospital Zurich, Switzerland

${ }^{2}$ Department of Pulmonology, University Hospital Zurich, Switzerland

${ }^{3}$ Department of Cardiovascular Surgery, University Hospital Zurich, Switzerland

${ }^{4}$ Institute of Pathology and Molecular Pathology, University Hospital Zurich, Switzerland

§ Isabelle Opitz and Olivia Lauk contributed equally as first authors

\section{Corresponding author:}

Beata Bode-Lesniewska

Institute of Pathology and Molecular Pathology

University Hospital

This article has been accepted for publication and undergone full peer review but has not been through the copyediting, typesetting, pagination and proofreading process, which may lead to differences between this version and the Version of Record. Please cite this article as doi: 10.1111/his.13773

This article is protected by copyright. All rights reserved. 
CH 8091 Zurich

Switzerland

beata.bode@usz.ch

Tel: +41442554051

Fax: +41442554416

Conflict of interest: The authors have no conflicts of interest.

\section{Contribution of the Authors:}

Study design: Isabelle Opitz, Olivia Lauk, Beata Bode-Lesniewska

Data acquisition and interpretation: Isabelle Opitz, Olivia Lauk, Didier Schneiter, Silvia Ulrich, Francesco Maisano, Walter Weder, Beata Bode-Lesniewska

Manuscript preparation: Isabelle Opitz, Olivia Lauk, Beata Bode-Lesniewska

Manuscript editing and Corresponding Author: Beata Bode-Lesniewska

Manuscript review and approval: All authors

Key words: sarcoma, pulmonary artery, EWSR1-CREB1 fusion, FISH, NGS, pulmonary endarterectomy

Fusions of the EWSR1 gene with partners from the CREB gene family have been identified in several histologic entities, mostly mesenchymal tumors of variable clinical malignant potential $^{1}$, including angiomatoid fibrous histiocytomas (AFH), clear cell sarcomas (CCS), clear cell sarcoma-like tumors of the gastrointestinal tract (CCSLTGT), primary pulmonary myxoid sarcoma $(\mathrm{PPMS})^{2}$, intracranial myxoid neoplasias $(\mathrm{iMN})^{3,4}$ and rare carcinoma variants. Interestingly, some of EWSR1-CREB-family gene fusion associated tumors show a prominent myxoid component and some of such tumors were histologically in the past

This article is protected by copyright. All rights reserved. 
diagnosed as "consistent with extraskeletal myxoid chondrosarcomas (EMC)" ${ }^{3,5}$. EMC is a rare sarcoma, currently defined as a myxoid mesenchymal tumor carrying the translocation of $N R 4 A 3$ gene with one of several partner genes ${ }^{6}, E W S R 1$ gene being one of the most common. Since EWSR1 gene is involved in the pathogenesis of aggressive Ewing sarcoma, EWSR1 FISH has been broadly available for some time for subtyping sarcomas. In the past, FISH evidence of the EWSR1 rearrangement in a myxoid mesenchymal neoplasm with compatible microscopic properties was often interpreted as being diagnostic for EMC. More recent findings, however, have proven, that some of these tumors do not belong to the EWSR1-NR4A3 category, but rather to the EWSR1-CREB family translocated tumor category, thus mandating caution in the interpretation of EWSR1 FISH in myxoid tumors. At present, the exact topographic distribution of myxoid mesenchymal EWSR1-CREB translocated tumors is not well known, due to the rarity of the entity as well as, the inherent diagnostic challenges. Recent reports have described such tumors as being mostly intrapulmonary (PPMS) ${ }^{2,5}$ or intracranial (iMN) ${ }^{3,4,7}$ with one perirectal example ${ }^{7}$. Hybrid tumors, with a typical AFH areas and a variable myxoid component have been described ${ }^{4,8}$, ${ }^{9}$ in a wide range of locations.

Intraluminal tumors of large vessels or the heart are rare. They correspond predominantly to undifferentiated pleomorphic sarcomas, designated as intimal sarcomas, showing amplification of the MDM2 gene ${ }^{10}$. There are rare reports on myxoid tumors in the large vessels or in the heart, mostly corresponding to pleomorphic sarcomas with focal myxoid change ${ }^{11}$. However, some of such tumors are referred to as of low-grade malignancy ${ }^{12,13}$. Molecular characterization in these reports is lacking. There is only one report on an intraluminal tumor of pulmonary artery morphologically corresponding to $\mathrm{AFH}$, which was found to contain EWSR1-ATF1 gene fusion ${ }^{14}$. In the current report, we describe a wellcharacterized, low grade myxoid, exclusively intraluminal tumor of the pulmonary artery with an EWSR1-CREB1 gene fusion and a long follow up.

This article is protected by copyright. All rights reserved. 
Histopathological material was retrieved from the pathology files of the Institute of Pathology, University Hospital, Zurich, Switzerland. Clinical and follow-up data were obtained from clinical databases of the Department of Thoracic Surgery. The study was approved by our institutional review board (the Cantonal Ethics Committee; KEK_ZH 2013_0430). Tumor tissue samples were fixed in buffered $4 \%$ formalin and embedded in paraffin. Immunohistochemistry (IHC) using the broad spectrum cytokeratin (AE1/AE3, Dako, Baar, Switzerland), SMA (1A4, Sigma, Darmstadt, Germany) and Mib1 (30-9, Ventana Roche, Basel, Switzerland) antibodies was performed using the Ventana Benchmark XT automated system (Ventana Medical Systems, Tucson, Arizona). Fluorescence In Situ Hybridization (FISH) studies were performed with dual color break-apart FISH detecting translocations of the EWSR1 (Vysis, Abbott AG, Baar, Switzerland) and NR4A3 (ZytoVision GmbH, Bremerhaven, Germany) genes as wells as dual color FISH detecting copy numbers of the centromeric region of the chromosome 12 and of the MDM2 gene for the evaluation of the amplification of the MDM2 gene (ZytoVision $\mathrm{GmbH}$, Bremerhaven, Germany) were performed. Next Generation Sequencing (NGS) of the tumor samples was performed as previously described with the commercially available Archer FusionPlex Sarcoma Panel (ArcherDx, Boulder, CO, USA), which simultaneously identifies fusions of 26 genes associated with soft tissue neoplasias ${ }^{15}$.

Clinical history: A 21-year old female presented with unspecific symptoms and deterioration in physical performance since few weeks. Laboratory work up disclosed elevated inflammatory parameters, thrombocytosis and anemia. Pulmonary function test showed significant restriction. Physical exam revealed abnormal systolic heart murmurs. Echocardiography showed pericardial fluid and a large mass in the pulmonary artery (PA). CT with angiography, MRI and ventilation-perfusion scan demonstrated a mass in the main trunk of the PA with slight metabolic uptake in the PET/CT scan (Figure 1 A). Endobronchial ultrasound-guided fine-needle aspiration contained abundant myxoid tumor tissue with low

This article is protected by copyright. All rights reserved. 
cellularity and the lack of necrosis or mitotic activity. Immunohistochemically, unspecific immunoprofile was seen, with weak expression of cytokeratin (AE1/AE3) (Figure 2 A) and no nuclear expression of MDM2 protein as well as a low proliferation rate (Figure $2 \mathbf{C}$ ). Cytopathological findings were diagnostic of a myxoid mesenchymal neoplasia, likely of lowgrade malignant potential. The findings were not diagnostic of an intimal sarcoma, as the features of a high-grade sarcoma were lacking. Following an interdisciplinary discussion, the decision was made to completely resect the tumor in curative intention, as there were no signs of metastatic disease. Pulmonary tumor endarterectomy (Figure 1 B, C) with reconstruction of the right PA in deep hypothermia was performed. The postoperative course was uneventful and the patient was discharged on the $6^{\text {th }}$ postoperative day. No adjuvant therapy was administered. The last regular follow up visit took place at 38 months after the first diagnosis, without clinical or imaging evidence of recurrence or metastatic disease. The patient has returned to her usual preoperative level of physical activity receiving lifelong anticoagulation. Several fragments of friable, myxoid, beige tumor tissue were submitted for histopathological analysis. Microscopically (Figure 2 A-C), abundant hypovascular, myxoid matrix was seen with focal hemosiderin deposition and a heterogeneous proliferation of middle-sized tumor cells, forming trabecular networks as well as rare solid areas. There was no lobulation, septation, inflammatory infiltration or necrosis. The tumor cells contained oval nuclei and a variable amount of eosinophilic cytoplasm, mostly with discernable cell borders. In selected solid areas, a more pronounced nuclear atypia was seen, which however was not accompanied by increased mitotic activity (Figure $2 \mathrm{C}$ ). Immunohistochemistry showed focal cytokeratin (AE1/AE3) (Figure 2 A), synaptophysin and SMA (Figure 2 B) expression, while reactions for EMA, S100, desmin, ERG, MDM2 and CDK4 were negative. Nuclear expression of INI1 protein was retained. The MiB1 proliferation index was low $(<10 \%)$. EWSR1 and MDM2 gene FISH analyses were performed at initial diagnosis and showed rearrangement of the EWSR1 gene (Figure 2 D), without amplification of the MDM2 gene (Figure 2 F). Rearrangement of the EWSR1 gene was considered compatible with the morphological diagnosis of EMC. Since molecular testing of the NR4A3 gene rearrangement 
was not available at initial diagnosis, the molecular background of the tumor could not be further clarified. Upon availability, NR4A3 FISH and NGS were performed retrospectively on the resected tumor specimen. No rearrangement of the $N R 4 A 3$ gene (Figure $2 \mathrm{E}$ ) was found on FISH; however, NGS analysis demonstrated a EWSR1-CREB1 fusion as the sole rearrangement.

Tumors of the pulmonary artery often show prominent or even predominant intraluminal growth in the form of clinically aggressive intimal sarcomas, that are often associated with amplifications of the $12 q 13-15$ chromosomal region ${ }^{10}$. Low-grade malignant tumors are less common in these locations ${ }^{12,13}$ and there are few data on their genetic background. In fact, there is only one report of a purely intraluminal primary tumor of a pulmonary artery in a 76 year-old woman, showing microscopical features of AFH and carrying the EWSR1-ATF1 gene fusion ${ }^{14}$. In the current study, we report on a purely myxoid, low-grade tumor with EWSR1-CREB1 fusion, confined to the lumen of the pulmonary artery in a 21-year old woman. Both ATF1 and CREB1 genes belong to the CREB family of transcription factors ${ }^{1}$. Fusions of EWSR1 with one of the genes of the CREB family occur in clinically heterogeneous, mostly mesenchymal neoplasia, comprising AFHs and other rare myxoid tumors, currently designated according to site of occurrence: tumors in the lung are referred to as "primary pulmonary myxoid sarcomas" (PPMS) ${ }^{2,5}$ and morphologically identical intracranial tumors as "intracranial myxoid neoplasm" (iMN) ${ }^{3,7,16}$. Only one such purely myxoid tumor outside of these two locations was reported in the perirectal region ${ }^{7}$. Even if non-specific, the immunoprofile of the current tumor does not fit well into the PPMS or myxoid AFH diagnostic categories, as the latter lack the expression of cytokeratins. Several earlier studies reported hybrid tumors containing gene fusions between EWSR1 and one of the genes of the CREB-family, so called "myxoid AFH" ${ }^{8,9}$, described both within the lung and intracranial locations $4,17,18$. Clinical data suggest that all three categories of the EWRS1-CREB family genes fusion carrying tumor types (pure $\mathrm{AFH}$, pure myxoid and hybrid

This article is protected by copyright. All rights reserved. 
AFH-myxoid variants) show low-grade malignant potential. Of the two pulmonary artery tumor with EWSR1-CREB family gene fusions, both were pure forms: an EWSR1-ATF1 AFH described by Ghinga ${ }^{14}$ and an exclusively myxoid tumor with EWSR1-CREB1 fusion from the current study. During the 38 months of follow up, there has been no evidence of local recurrences or metastases in our patient, while follow up data are not provided for the patient with the previously described AFH. Since there are no descriptions of hybrid forms in this location, it remains unclear, weather these two tumors are in fact within the spectrum of the same entity.

Initial histopathological evaluation of the present case pointed out towards the diagnosis of an EMC. In view of the increased availability of molecular tools for routine diagnostics in recent years, reevaluation of the diagnoses of soft tissue and bone tumors is often warranted $^{19}$. Currently, histopathologic diagnosis of EMC is based on the demonstration of a rearrangement of the $N R 4 A 3^{6}$. This genetic aberration has only become recently available for routine diagnostics, so that the EMC diagnosis was considered one of exclusion, in some cases supported by the demonstration of the rearrangement of the EWSR1 gene (as in our case). In our case, extended FISH and NGS analyses demonstrated the lack of the rearrangement of the $N R 4 A 3$ gene and the CREB1 gene was identified as the fusion partner of the rearranged EWSR1 gene, removing the tumor from the category of the EMC. Morphologically comparable tumor, diagnosed as an EMC was described in $2010^{20}$ in the heart of a 26-year-old man, however the tumor disease was widely metastatic, so contrary to our case, it remains unresolved if this is a primary or metastatic heart tumor. The EWSR1 gene $\mathrm{FISH}$ in this case was negative and no further molecular studies were done. Yet another tumor reported as an EMC of $\mathrm{PA}^{21}$ is obviously incorrectly diagnosed as such, since no molecular studies were performed and the microphotographs illustrate hyaline chondrogenic neoplasia, immunohistochemically strongly expressing S100 protein, findings excluding the diagnosis of EMC.

This article is protected by copyright. All rights reserved. 
In conclusion we describe a well-documented tumor with an EWSR1-CREB1 fusion, which has never previously been reported in a purely intraluminal tumor of large vessels or heart. This report widens the spectrum of possible distributions of EWSR1-CREB-family rearranged myxoid tumors on one hand, and the spectrum of differential diagnoses of primary intravascular neoplasias of large vessels on the other.

\section{Legends}

Figure 1: (A) PET-CT showed slight metabolic activity of the intraluminal pulmonary artery mass (arrow) (B, C) Intraoperativelly, a gelatinous tumor (asterisk), freely floating in the vessel and attached to the left pulmonary artery wall was found and completely resected.

Figure 2: (A) Intraluminal tumor mass of the pulmonary artery consisted of polypoid tumor tissue with predominantly low cellularity (H\&E; 50x) and focal cytokeratin AE1/AE3 expression (inset; 400x). (B) Middle sized spindle cells were embedded in prominent myxoid extracellular matrix and showed a partial trabecular growth pattern (H\&E; 400x) with focal SMA expression (inset; 400x). (C) Focal increased cellularity with mild atypia could be observed, however the proliferation rate was low (inset, Mib1; 400x). (D) Break apart FISH of the EWSR1 gene showed one fused signal (arrowhead) and one split pair of red and green signals (arrows) in the nuclei of the tumor cells, indicating rearrangement of the EWSR1 gene. (E) Tumor cell nuclei in the break apart FISH analysis of the NR4A3 gene contained exclusively fused signals (arrowheads), suggesting absence of the rearrangement of the NR4A3 gene. (F) No amplification of the MDM2 gene was observed in the FISH study.

This article is protected by copyright. All rights reserved. 


\section{References:}

1. Thway K, Fisher C. Tumors with ewsr1-creb1 and ewsr1-atf1 fusions: The current status. Am J Surg Pathol 2012;36;e1-e11.

2. Thway K, Nicholson AG, Lawson $\mathrm{K}$ et al. Primary pulmonary myxoid sarcoma with ewsr1-creb1 fusion: A new tumor entity. Am J Surg Pathol 2011;35;1722-1732.

3. Velz J, Agaimy A, Frontzek K et al. Molecular and clinicopathologic heterogeneity of intracranial tumors mimicking extraskeletal myxoid chondrosarcoma. J Neuropathol Exp Neurol 2018;77;727-735.

4. Bale TA, Oviedo A, Kozakewich $\mathrm{H}$ et al. Intracranial myxoid mesenchymal tumors with ewsr1-creb family gene fusions: Myxoid variant of angiomatoid fibrous histiocytoma or novel entity? Brain Pathol 2018;28;183-191.

5. Matsukuma S, Hisaoka M, Obara $\mathrm{K}$ et al. Primary pulmonary myxoid sarcoma with ewsr1-creb1 fusion, resembling extraskeletal myxoid chondrosarcoma: Case report with a review of literature. Pathology International 2012;62;817-822.

6. Lucas DR, Stenman G. Extraskeletal myxoid chondrosarcoma. In Fletcher CDM, Bridge JA, Hogendoorn PCW, Mertens F eds. Who classification of tumours of soft tissue and bone. Lyon: IARC, 2013;223-224.

7. Kao YC, Sung YS, Zhang $L$ et al. Ewsr1 fusions with creb family transcription factors define a novel myxoid mesenchymal tumor with predilection for intracranial location. Am J Surg Pathol 2017;41;482-490.

8. Schaefer IM, Fletcher CD. Myxoid variant of so-called angiomatoid "malignant fibrous histiocytoma": Clinicopathologic characterization in a series of 21 cases. Am J Surg Pathol 2014;38;816-823.

9. Chen G, Folpe AL, Colby TV et al. Angiomatoid fibrous histiocytoma: Unusual sites and unusual morphology. Modern Pathology 2011;24;1560.

10. Bode-Lesniewska B, Debies-Rychter M. Intimal sarcoma. In Fletcher CDM, Bridge JA, Hogendoorn PCW, Mertens F eds. Who classification of tumours of soft tissue and bone. Lyon: IARC, 2013;232-233.

11. Burke A, Tavora FR, Maleszewski JJ, Frazier AA. Tumors of the heart and great vessels. Silver Spring, Maryland: American Registry of Pathology, 2015.

12. Suh JH, Kim DY, Yoon JS, Park ES, Park CB. Low grade myxofibrosarcoma in the right ventricle presenting as pulmonary thromboembolism. $J$ Thorac Dis 2017;9;E1084E1087.

13. Gupta $\mathrm{P}$, Jain M. Right-sided low grade myxofibrosarcoma of the heart in an adult. Asian Cardiovascular and Thoracic Annals 2013;21;208-210.

14. Ghigna M-R, Hamdi S, Petitpretz $P$ et al. Angiomatoid fibrous histiocytoma of the pulmonary artery: A multidisciplinary discussion. Histopathology 2014;65;278-282.

15. Velz J, Agaimy A, Frontzek K et al. Molecular and clinicopathologic heterogeneity of intracranial tumors mimicking extraskeletal myxoid chondrosarcoma. Journal of Neuropathology \& Experimental Neurology 2018;77;727-735.

16. Sciot R, Jacobs S, Calenbergh FV, Demaerel P, Wozniak A, Debiec-Rychter M. Primary myxoid mesenchymal tumour with intracranial location: Report of a case with a ewsr1-atf1 fusion. Histopathology 2018;72;880-883.

17. Gareton A, Pierron G, Mokhtari K et al. Eswr1-crem fusion in an intracranial myxoid angiomatoid fibrous histiocytoma-like tumor: A case report and literature review. Journal of Neuropathology \& Experimental Neurology 2018;77;537-541.

18. Smith SC, Palanisamy N, Betz BL et al. At the intersection of primary pulmonary myxoid sarcoma and pulmonary angiomatoid fibrous histiocytoma: Observations from three new cases. Histopathology 2013;65;144-146.

19. Noujaim J, Jones RL, Swansbury J et al. The spectrum of ewsr1-rearranged neoplasms at a tertiary sarcoma centre; assessing 772 tumour specimens and the value of current ancillary molecular diagnostic modalities. Br J Cancer 2017;116;669-678.

This article is protected by copyright. All rights reserved. 
20. Geyer HL, Karlin N. Extraskeletal myxoid chondrosarcoma of the heart and review of current literature. Curr Oncol 2010;17;58-62.

21. Gadabanahalli K, Belaval VV, Bhat V, Gorur IM. Primary extraskeletal myxoid chondrosarcoma of pulmonary arteries: A rare mimic of acute pulmonary thromboembolism. Interact Cardiovasc Thorac Surg 2015;20;565-566.

This article is protected by copyright. All rights reserved. 

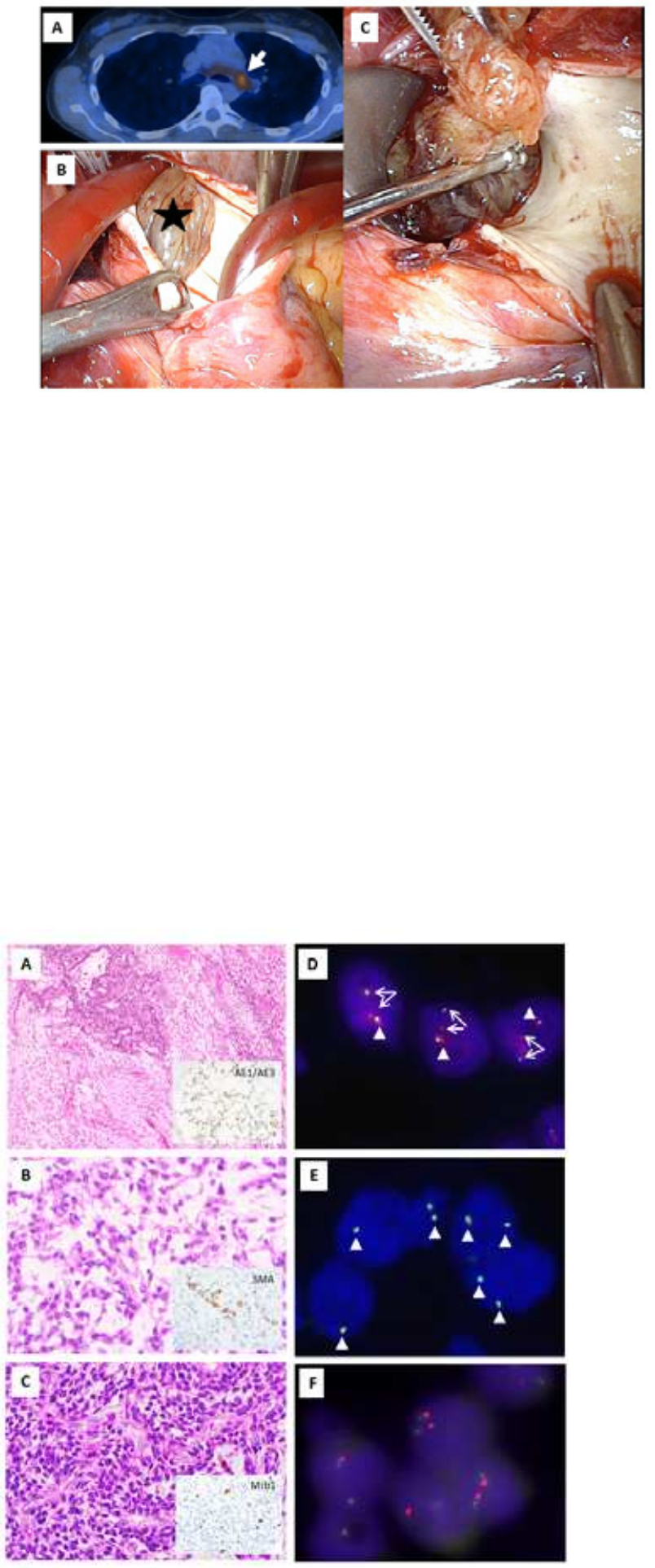

This article is protected by copyright. All rights reserved. 